\title{
Consequences of the Complex Character of the Internal Symmetry in Supersymmetric Theories *
}

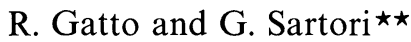 \\ Département de Physique Théorique, Université de Genève, CH-1211 Genève 4, \\ Switzerland
}

\begin{abstract}
The consequences of the invariance of the superpotential under the complexification $G^{c}$ of the internal symmetry group on the determination of the possible patterns of symmetry and supersymmetry breaking are established in a globally supersymmetric theory. In particular, in the case of global internal symmetry we show that a vacuum associaated to a point $z$, where $G_{z}{ }^{c} \neq G_{z}^{c}$ is always degenerate with a vacuum associated to a point $z^{\prime}$, where $G_{z^{\prime}}^{c}=G_{z^{\prime}}{ }^{c}$; all the other degeneracies of the minimum of the potential on an orbit of $G^{c}$ are also determined and shown to be completely removed when the internal symmetry is gauged. The zeroes of the $D$-term of a supersymmetric gauge theory are characterized as the points of the closed orbits of $G^{c}$ which are at minimum distance from the origin; at these points $G_{z}^{c}=G_{z}{ }^{c}$. It is rigorously proved that the minimum of the potential is zero if the gradient of the superpotential vanishes somewhere. It is also shown that the $D$-term necessarily vanishes at the minimum of the potential if the direction of spontaneous supersymmetry breaking is invariant by $G$.
\end{abstract}

\section{Introduction}

Rigid supersymmetric theories, exhibiting additional global or local internal symmetries, have been the object of considerable interest lately [1]. The interest has survived the advent of local supersymmetry, as it is reasonable to believe that the breaking of supergravity takes place at very high scale such that the effective low energy theory is indeed a rigid supersymmetry, spontaneously broken or broken by explicit soft terms.

The possible patterns of supersymmetry and/or internal symmetry breaking are contained in the form of the superpotential of a supersymmetric theory. It has been remarked by many authors [2] that the complexification $G^{c}$ of the compact internal symmetry group $G$ may play an important role in this context,

\footnotetext{
* Partially supported by the Swiss National Science Foundation and INFN, Sezione di Padova $\star \star$ On leave of absence from the Department of Physics of the University of Padova, Italy
} 
particularly, if the local and global geometries of its orbits are analyzed [3-5]. In the second part of the present paper we shall essentially follow the approach of [3] with the purpose of examining how the possible patterns of supersymmetry and internal-symmetry breaking are constrained by the particular way in which $G$ invariance is realized in the potential of a globally supersymmetric theory. The derivation of new results has been possible in part because of recent progress in related mathematics, which we shall try to summarize in accessible terms in the first part of the paper. A considerable part of the present paper is reserved for the presentation of mathematical concepts, not used in physics so far, in the conviction that they may prove of some usefulness in the future development of the subject.

The mathematical preliminaries contained in the four sections of Part I of the paper are only partially due to ourselves. The rest is taken from specialized textbooks and mathematical literature, and has been freely manipulated in order to be directly exploitable in the solution of problems of physical interest. As a rule, the proofs of those results which we could find in textbooks will not be reproduced (see for instance [6-9]). Some other results will be proved in Appendix A with the constant sacrifice of generality in favour of simplicity.

Part II of the paper, devoted to physical applications in the framework of globally supersymmetric theories, is divided into four sections. After a short introduction in Sect. II.1, in Sect. II.2 we will determine all those degeneracies of the vacuum state that are related to the particular way in which the $G$-invariance is realized in the effective potential when the internal symmetry is global.

In Sect. II.3 we shall be concerned with local internal symmetries. The main effort will be put in characterizing the situations in which the vacuum state is associated to a zero of the $D$-term of the effective potential. We shall prove theorems which give a hint towards understanding why the minima of the effective potentials are so frequently associated to zeros of the $D$-term.

In Sect. II.4 we shall illustrate the ways in which our results can be adapted to the case of a partially gauged internal symmetry.

\section{Mathematical Preliminaries}

\section{Complexification of a Compact Lie Group}

Throughout the paper $G$ will denote a compact Lie group of unitary transformations acting linearly on the vectors $z=\left(z_{1}, \ldots, z_{n}\right)$ of an $n$-dimensional complex vector space $V \simeq C^{n}$. The connected component of $G$ ill be denoted by $G_{0}$ and $K$ will denote the finite group $G / G_{0}$.

Below we shall define the complexification $G^{c}$ of $G$ and some elementary properties of the isotropy subgroups of the related linear action.

The elements of the Lie algebra $\mathscr{G}$ of $G$ are antihermitian matrices and will be denoted by $t$ :

$$
t^{+}=-t, \quad t \in \mathscr{G}
$$

When necessary we shall introduce in $\mathscr{G}$ a basis $\left\{t_{\alpha}\right\}_{1 \leqq \alpha \leqq r}$, orthonormal with respect to the Killing form:

$$
\operatorname{tr}\left(\operatorname{adj} t_{\alpha} \cdot \operatorname{adj} t_{\beta}\right)=-\delta_{\alpha \beta}, \quad \alpha=1, \ldots, r
$$


The complexification $\mathscr{G}^{c}$ of $\mathscr{G}$ is obtained by extending the basic field of $\mathscr{G}$ from the real to the complex numbers:

$$
\mathscr{G}^{c}=\left\{\tau \mid \tau=t+i t^{\prime} ; t, t^{\prime} \in \mathscr{G}\right\} .
$$

A basis for $\mathscr{G}$ is evidently also a basis for $\mathscr{G}^{c}$. The Lie algebra $\mathscr{G}^{c}$, originally defined as an algebra on the complex numbers, can be also considered as a $2 r$ dimensional real Lie algebra (also denoted by the same symbol). In this case a basis for $\mathscr{G}^{c}$ is for instance $\left\{t_{\alpha}, \tilde{t}_{\alpha}\right\}_{1 \leqq \alpha \leqq r}$, where $\tilde{t}_{\alpha}=i t_{\alpha}$. Clearly:

$$
\operatorname{dim}_{R} \mathscr{G}^{c}=2 \operatorname{dim}_{c} \mathscr{G}^{c}=2 \operatorname{dim}_{R} \mathscr{G}=2 r .
$$

As a real Lie algebra, $\mathscr{G}^{c}$ generates a $2 r$-dimensional connected Lie group $G_{0}{ }^{c}$ which is called the complexification of $G_{0}$. The group $G^{c}=K G_{0}{ }^{c}$, is by definition, the complexification of $G$.

In the following we shall use latin symbols to denote elements of $\mathscr{G}$ and $G$ and greek ones to denote elements of the complexifications.

Both $\mathscr{G}^{c}$ and $G^{c}$ are closed with respect to hermitian conjugation:

$$
\tau^{+}=-t+i t^{\prime} \in \mathscr{G}^{c}, \quad \forall \tau=t+i t^{\prime} \in \mathscr{G}^{c} ; \quad t, t^{\prime} \in \mathscr{G}, \quad \gamma^{+} \in G^{c}, \quad \forall \gamma \in G^{c} .
$$

The group $G^{c}$ is an algebraic subgroup of $\operatorname{GL}(n, C)$, that is a subgroup of $\mathrm{GL}(n, C)$ formed by the matrices which satisfy a set of convenient polynomial relations:

$$
q_{a}(\gamma)=0, \quad a=1, \ldots, A .
$$

The group $G^{c}$ is not compact and $G$ is one of its maximal compact subgroups, so that every compact subgroup of $G^{c}$ is conjugated in $G^{c}$ to a compact subgroup of $G$.

Every representation of $G$ can be extended by complexification to a representation of $G^{c}$ and every (rational) representation of $G^{c}$ can be obtained in this way.

Since all the representations of a compact group are completely reducible, the same will be true of the representations of $G^{c}$.

An algebraic group whose representations are all completely reducible is said to be reductive. So $G^{c}$ is a reductive algebraic group. It can be shown that:

$\mathbf{P 1}$ [6]. A complex linear algebraic group is reductive if and only if it is the complexification of one of its maximal compact subgroups.

The reductivity of a linear algebraic group is equivalent to that of its Lie algebra.

Isotropy subgroups and subalgebras at $z$ will be denoted by $G_{z}, G_{z}^{c}, \mathscr{G}_{z}, \mathscr{G}_{z}^{c}$. By definition:

$$
\begin{array}{lll}
g \cdot z=z=\gamma \cdot z, & \forall g \in G_{z}, & \gamma \in G_{z}^{c}, \\
t \cdot z=0=\tau \cdot z, & \forall t \in \mathscr{G}_{z}, & \tau \in \mathscr{G}^{c}{ }_{z} .
\end{array}
$$

If $\tau=t+i t^{\prime}$ and $t, t^{\prime} \in \mathscr{G}_{z}$, then $\tau \cdot z=0$ and consequently:

$$
\mathscr{G}_{z}{ }^{c} \subseteq \mathscr{G}^{c}{ }_{z}, \quad G_{z}{ }^{c} \subseteq G^{c}{ }_{z},
$$

where $\mathscr{G}_{z}{ }^{c}$ denotes the complexification of $\mathscr{G}_{z}$. 
Since $\tau \cdot z=0$ does not generally imply $t \cdot z=0=t^{\prime} \cdot z$, the equality sign in Eq. (1.9) is exceptional. ${ }^{1}$ It will also be useful to reformulate our last remark in the following way: since $t \cdot z=0=t^{\prime} \cdot z$ if and only if $\tau \cdot z=0=\tau^{+} \cdot z$, and by definition $\mathscr{G}_{z}{ }^{c}=\left(\mathscr{G}_{z}{ }^{c}\right)^{+}$, the subalgebra $\mathscr{G}_{z}{ }^{c}$ can be characterized as the maximal Lie subalgebra of $\mathscr{G}^{c}{ }_{z}$ which is closed with respect to hermitian conjugation. Thus:

$$
\operatorname{dim}_{R} \mathscr{G}_{z}^{c} \geqq \operatorname{dim}_{R} \mathscr{G}_{z}^{c}=2 \operatorname{dim}_{R} \mathscr{G}_{z} .
$$

Example 1. $G=S U_{2}$ in $C^{2}, G^{c}=S L_{2}(C)$. Isotropy subgroups: every non-null vector $z=\left(z_{1}, z_{2}\right)$ can be transformed by means of a $S L_{2}(C)$ transformation into $w=(1,0)$ and

$$
G_{w}=\{1\}=G_{w}{ }^{c} \neq G_{w}^{c}=\left\{\left(\begin{array}{ll}
1 & c \\
0 & 1
\end{array}\right)\right\}_{c \in C^{1}} .
$$

With reference to a given linear group the isotropy subgroups at points of the same orbit are conjugated. Thus:

$$
\begin{array}{cc}
G_{\gamma \cdot z}^{c}=\gamma G_{z}^{c} \gamma^{-1}, & \gamma \in G^{c} ; \\
G_{g \cdot z}=g G_{z} g^{-1}, & g \in G ;
\end{array}
$$

but it is easy to realize that there is generally no simple relation between $G_{\gamma \cdot z}$ and $G_{z}$ when $\gamma \in\left(G^{c}-G\right)$, as shown in the following example.

Example 2. $G=S_{3}(R)$ acting in $C^{3}, G^{c}=S O_{3}(C)$. The two points $u=(1,0,0)$ and $v=(\cos w, \sin w, 0), w \in C^{1}, \operatorname{Re} w \operatorname{Im} w \neq 0$, are on the same $G^{c}$-orbit, but it is easy to check that:

$$
G^{c}{ }_{u} \simeq S O_{2}(C) \simeq G_{v}^{c}, \quad G_{u} \simeq S O_{2}(R) ; \quad G_{v} \simeq\{1\} .
$$

Since all the points of the same orbit have conjugated isotropy subgroups, the class $\left(G_{z}\right)$ of all the subgroups of $G$ conjugated to $G_{z}$ will be called the "symmetry of the $G$-orbit through $z$." Analogously, one will define the symmetry of a $G^{c}$-orbit. In formulas:

$$
\left(G_{z}\right)=\left\{g G_{z} g^{-1}\right\}_{g \in G} ; \quad\left(G_{z}^{c}\right)=\left\{\gamma G_{z}^{c} \gamma^{-1}\right\}_{\gamma \in G^{c}} .
$$

The group $G_{z}^{c}$ is clearly algebraic, but not necessarily reductive. In Example 1, for instance, the linear group $G_{w}^{c}$, which yields a representation of the additive group of complex numbers, is not reductive. In fact, the linear group itself is reducible, since $C^{2}$ contains a $G^{c}{ }_{w}$-stable subspace $V_{1}=\{\lambda w\}_{\lambda \in C^{1}}$, but it is not completely reducible, since no complementary subspace to $V_{1}$ in $C^{2}$ is left invariant by $G^{c}$.

\section{Closed Orbits (and Vectors of Minimal Length)}

Closure properties of $G^{c}$-orbits will play a central role in the physical applications we shall describe in the second part of the paper. Therefore, we shall devote the present section to a characterization of this distinguishing topological property and of its implications.

${ }^{1}$ The fact that $G^{c}{ }_{w}$ is in general different from $G_{w}{ }^{c}$ was remarked in [3], in connection with the problem of characterizing the zeroes of the $D$-term (see also $[4,5]$ ). The relation $G_{w}{ }^{c}=G^{c}{ }_{w}$ is also relevant to the phenomenon of exact doubling of the Goldstone bosons (see [10]). The problem is discussed for instance in [11] 
Owing to the Lie structure of $G$ and $G^{c}$, both $G$ - and $G^{c}$-orbits are smooth submanifolds of $C^{n}$ [7]. Since $G$ is compact, $G$-orbits are compact subsets of $C^{n}$, and therefore bounded and closed subsets. The same is not true, in general of $G^{c}$ orbits; however, from Theorem 8.3 of [7] one can easily derive that:

P2. The closure $\bar{\Omega}$ of every $G^{c}$-orbit $\Omega$ is an algebraic subset of $C^{n}$.

By algebraic set in $C^{n}$ is meant the set of all the common zeroes of a set of complex polynomial functions defined on $C^{n}$.

The closure of a $G^{c}$-orbit can be tested by means of a fundamental criterium, due to Hilbert and Mumford [9], which will be stated, without proof and in a form suitable to our purposes, under item P3.

P3. The $G^{c}$-orbit $\Omega$ is closed if and only if there is no one-parameter subgroup $\Gamma$ of $G^{c}, \Gamma=\left\{e^{a t}\right\}_{a \in R^{1}}, i t \in \mathscr{G}$ such that the limit $\lim _{a \rightarrow \infty} e^{a t} \cdot z$, exists and does not belong to $\Omega$.

Example 3. $G=U_{1}$ acting in $C^{1}, G^{c}=G L_{1}(C)$. There are only two orbits:

1. the origin $z=0$, which is a closed subset;

2. the rest of the complex plane, which is clearly an open set.

Example 4. $G=U_{1}$ acting in $C^{2}, G^{c} \simeq G L_{1}(C)$ :

$$
g=\left(\begin{array}{ll}
e^{i b} & \\
& e^{-i b}
\end{array}\right), \quad \gamma=\left(\begin{array}{ll}
e^{a+i b} & \\
& e^{-(a+i b)}
\end{array}\right), \quad a, b \in R^{1} .
$$

There are three types of orbits: 1 . the point $z=0$ (closed); 2. orbits through points $z=\left(z_{1}, z_{2}\right) \neq 0$ with $z_{1} z_{2}=0$ (non-closed); 3 . orbits through generic points $z=\left(z_{1}, z_{2}\right), z_{1} z_{2} \neq 0$ (closed).

Orbits of type 2 have evidently zero in their boundary and:

$$
\lim _{a \rightarrow \infty}\left(\begin{array}{cc}
e^{a} & \\
& e^{-a}
\end{array}\right)\left(\begin{array}{c}
0 \\
z_{2}
\end{array}\right)=0=\lim _{a \rightarrow+\infty}\left(\begin{array}{cc}
e^{-a} & \\
& e^{a}
\end{array}\right)\left(\begin{array}{c}
z_{1} \\
0
\end{array}\right) .
$$

On the contrary it is quite evident that, starting from points lying on orbits of type 3 , no limit on the action of a one parameter subgroup of $G L_{1}(C)$ can eventually lead to a finite point outside the orbit.

Example 5. $G$ is a unitary $\underline{3}+\underline{8}$, matrix representation of $S U_{3}$. Let $g \in G$ be the representative of $u \in S U_{3}$. The eleven dimensional complex vector $z$ can be considered as a direct sum of a three dimensional complex vector $w$ and a traceless $3 \times 3$ matrix $\lambda$, on which $g \in G$ acts in the following way: $g \cdot(w, \lambda)=\left(u w, u \lambda u^{-1}\right)$.

The complexification of $G$ leads to a $\underline{3}+\underline{8}$ representation of $S L_{3}(C)$. The representative $\gamma$ of an element $a \in S L_{3}(C)$ acts on $z=(w, \lambda)$ in the following way: $\gamma \cdot(w, \lambda)=\left(a w, a \lambda a^{-1}\right)$.

Let us consider the $G^{c}$-orbit through $z_{0}=\left(w_{0}, \lambda_{0}\right), \quad w_{0}=(1,0,0)$, $\lambda_{0}=\operatorname{diag}(-2,1,1)$. It is not closed, as one easily checks using the Hilbert-Mumford criterium. In fact: $\lim _{x \rightarrow+\infty} e^{x \lambda_{0}} \cdot z_{0}=\left(0, \lambda_{0}\right) \notin G^{c} \cdot z_{0}$.

The isotropy subgroup of $G^{c}$ at $z_{0}$ is formed by the representatives in $G^{c}$ of those matrices of $S L_{3}(C)$ which commute with $\lambda_{0}$, and leave $w_{0}$ stable. Therefore, $a \in S L_{3}(C)$ has representatives in $G_{z_{0}}^{c}$ if and only if $a=\left(\begin{array}{c|c}1 & 0 \\ \hline 0 & \bar{a}\end{array}\right)$, where $\hat{a} \in S L_{2}(C)$, 
so that $G_{z_{0}}^{c} \simeq S L_{2}(C)=\left(S U_{2}\right)^{c}$. The same argument leads to $G_{z_{0}} \simeq S U_{2}$, so that in this particular case $G_{z_{0}}^{c}$ is reductive. As we shall see this last property is quite exceptional for non-closed orbits, while it is the rule for closed ones.

Owing to P2, the boundary $\bar{\Omega}-\Omega$ of a $G^{c}$-orbit $\Omega$ is an algebraic set of lower dimensions than $\Omega$; moreover, it is easy to realize that the closure of $\Omega$ is a $G^{c}$-stable set: $\gamma \cdot z \in \bar{\Omega}$ for all $z \in \bar{\Omega}$ and $\gamma \in G^{c}$. Therefore:

P4 [7, Proposition 8.3]. The boundary of every $G^{c}$-orbit $\Omega, \bar{\Omega}-\Omega$, is a union of $G^{c}$ orbits (not necessarily closed) of lower dimensions.

By iterating the argument leading to $\mathbf{P 4}$ one concludes that in the closure of every $G^{c}$-orbit there is at least one closed $G^{c}$-orbit (in fact, it is unique as we shall see):

P5 [7, Proposition 8.3]. Every lowest-dimension $G^{c}$-orbit, contained in the closure of a $G^{c}$-orbit, is a closed orbit.

Below, under items P6, P7, and P8 we shall characterize the closed $G^{c}$-orbits in a way which will be interesting for physical applications.

If $G$ is a group of unitary transformations of $C^{n}$, its orbits are formed by vectors of constant length. The same is certainly not true of the $G^{c}$-orbits, along which one can generally reach infinity. Are there "vectors of minimum length" on a $G^{c}$-orbit? It is easy to realize that the answer is positive if the orbit is closed. In fact, a closed set always admits points at minimum distance from a given point $(z=0)$ in $C^{n}$. For non-closed orbits, which are generally not even open, the answer is not trivial, and has been given by Kempf and Ness [12]. In order to report their results, let us denote by $\left.f(z)\right|_{\Omega}$ the restriction of the function $f(z)$ to the $G^{c}$-orbit $\Omega$. Then:

P6 [10]. The function $\left.\|z\|^{2}\right|_{\Omega}$ takes on its absolute minimum at all its stationary points. that:

A simple proof of P6 will be given in Appendix A where we shall also show

P7 [10]. On a $G^{c}$-orbit there are vectors of minimum length if and only if the orbit is closed.

If $z$ is a vector of minimum length ${ }^{2}$, then all the vectors forming the $G$-orbit through $z$ share the same property. Kempf and Ness have shown that they are the only ones with such a property (for a proof see Appendix A).

P8 [12]. The vectors of minimum length on a $G^{c}$-orbit form a unique G-orbit.

The following result, first remarked in [3] (for a proof see Appendix A) points out an important property of the isotropy subgroup of $G^{c}$ at vectors of minimum length. As we shall see in Part II, it has significant applications in supersymmetric gauge theories for the constraints it puts on the mass spectrum.

P9. If $z$ is a vector of minimum length, the isotropy subgroup of $G^{c}$ at $z, G^{c}$, coincides with the complexification $G_{z}{ }^{c}$ of $z$.

\footnotetext{
${ }^{2}$ In the rest of the paper, "vector $z$ of minimum length" will stay for "vector $z$ of minimum length on the $G^{c}$-orbit through $z$ "
} 
The claim under the following item P10 can be derived as an immediate corollary to $\mathbf{P 9}$ and $\mathbf{P 8}$ :

P10. The symmetry of closed $G^{c}$-orbits is reductive [13] and on every closed $G^{c}$-orbit there is at least one $G$-orbit $\mathcal{O}$, where $G^{c}{ }_{z}=G_{z}{ }^{c}$, for all $z \in \mathcal{O}$.

The converse of $\mathbf{P 1 0}$ is not generally true, as we have just seen, in Example 5: the reductivity of the symmetry of an orbit does not imply its closure. ${ }^{3}$ An even simpler example is yielded by the linear group examined in Example $4\left(G^{c}=\right.$ the multiplicative group of complex numbers acting in $C^{2}$ ). There, for every $z=\left(z_{1}, z_{2}\right) \neq 0$ of $C^{2}$ one gets: $G_{z}^{c}=\{1\}=G_{z}{ }^{c}$, showing that the isotropy subgroup of $G^{c}$ at $z$ is the same both for $z_{1} z_{2} \neq 0$ (in which case $z$ lies on a closed orbit) and for $z_{1} z_{2}=0$ (non-closed orbit).

Even if there are embarassingly simple counterexamples to the converse of P1, they correspond to rather exceptional situations.

A theorem due to Luna [15], which we shall mention without proof under item P11, will shed some further light on the relation between the closure properties of an orbit and the reductivity of its symmetry. Let us call $N^{c}(K)$ the normalizer in $G^{c}$ of a subgroup $K$ of $G^{c}$. The subgroup $N^{c}(K)$ is formed by the elements $\gamma$ of $G^{c}$ satisfying the relation $\gamma K^{-1}=K$. As is well known, $K$ is an invariant subgroup of its normalizer.

P11 [15]. Let $K$ be a reductive algebraic subgroup of $G^{c}$, then the following conditions $i)$ and ii) are equivalent:

i) $N^{c}(K) / K$ is a finite group;

ii) in all finite dimensional rational representations of $G^{c}$, all $G^{c}$-orbits whose symmetry is $\supseteqq(K)$ are closed.

\section{Local Structure of an Orbit Near Vectors of Minimum Length}

In this section we shall report results proved in [3] concerning the local structure of a closed $G^{c}$-orbit near vectors of minimum length. We shall first define the "realification" of the linear action of $G^{c}$.

The natural correspondence $C^{n} \leftrightarrow R^{2 n}$, set by the relation:

$$
C^{n} \ni z \leftrightarrow \zeta=(\operatorname{Re} z, \operatorname{Im} z) \in R^{2 n},
$$

induces a linear action of $G^{c}$ in $R^{2 n}$ defined, for all $\gamma \in G^{c}$, by:

$$
\gamma \cdot \zeta=\left(\begin{array}{c|r}
\operatorname{Re} \gamma & -\operatorname{Im} \gamma \\
\hline \operatorname{Im} \gamma & \operatorname{Re} \gamma
\end{array}\right)\left(\begin{array}{l}
\operatorname{Re} z \\
\operatorname{Im} z
\end{array}\right) .
$$

The restriction to $G$ of this action is real orthogonal and the representation of the Lie algebra $\mathscr{G}$ associated to its induced action in $R^{2 n}$ :

$$
t \cdot \zeta=\left(\begin{array}{r|r}
\operatorname{Re} t & -\operatorname{Im} t \\
\hline \operatorname{Im} t & \operatorname{Re} t
\end{array}\right) \cdot\left(\frac{\operatorname{Re} z}{\operatorname{Im} z}\right)
$$

is real antisymmetric.

\footnotetext{
${ }^{3}$ On this point we disagree with the authors of [4]. The proof of their theorem is spoilt by the use of a wrong statement erroneously attributed to the author of [12]
} 
The multiplication by $\sqrt{-1}$ in $C^{n}$ is represented in $R^{2 n}$ by the action of the following operator $\varepsilon$ :

$$
\varepsilon=\left(\begin{array}{rr}
0 & -1 \\
1 & 0
\end{array}\right)
$$

which commutes with the action of every $\gamma \in G^{c}$ :

$$
(\varepsilon \cdot \gamma-\gamma \cdot \varepsilon) \cdot \zeta=0, \quad \forall \gamma \in G^{c}, \quad \zeta \in R^{2 n} .
$$

The standard hermitian scalar product (, ) of $C^{n}$ can be calculated in terms of the matrix $\varepsilon$ and the euclidean scalar product $\langle$,$\rangle of R^{2 n}$ :

$$
\left(z, z^{\prime}\right)=\sum_{1}^{n} z_{k}{ }_{k} z^{\prime}=\left\langle\zeta, \zeta^{\prime}\right\rangle+i\left\langle\varepsilon \zeta, \zeta^{\prime}\right\rangle .
$$

The $G$ - and $G^{c}$-orbits in $R^{2 n}$ are smooth submanifolds of $R^{2 n}$ and the corresponding tangent spaces at $\zeta$ are, respectively, $\zeta+T_{\zeta}$ and $\zeta+T_{\zeta}^{c}$, where $T_{\zeta}$ and $T_{\zeta}^{c}$ are the spaces formed by all the vectors $t \cdot \zeta, t \in \mathscr{G}$ and, respectively, $\tau \cdot \zeta, \tau \in \mathscr{G}^{c}$ :

$$
T_{\zeta}=\{t \cdot \zeta \mid t \in \mathscr{G}\} ; \quad T_{\zeta}^{c}=\left\{\tau \cdot \zeta \mid \tau \in \mathscr{G}^{c}\right\} .
$$

In the following $T_{\zeta}$ and $T_{\zeta}^{c}$ will be briefly called tangent spaces to the $G$ - and $G^{c}$ orbits at $\zeta$.

The orthogonal spaces to the $G$ - and $G^{c}$-orbits at $\zeta$ will be denoted by $N_{\zeta}$ and $N_{\zeta}^{c}$, respectively, and defined as the orthogonal complements of $T_{\zeta}$ and $T_{\zeta}^{c}$ in $R^{2 n}$ :

$$
\begin{aligned}
N_{\zeta} & =\left\{\xi \in R^{2 n} \mid\langle\xi, t \cdot \zeta\rangle=0, \forall t \in \mathscr{G}\right\}, \\
N_{\zeta}^{c} & =\left\{\xi \in R^{2 n} \mid\langle\xi, \tau \cdot \zeta\rangle=0, \forall \tau \in \mathscr{G}^{c}\right\} .
\end{aligned}
$$

The one-to-one correspondence between points of $C^{n}$ and $R^{2 n}$ set in Eq. (3.1) is a $G^{c}$-invariant homeomorphism, mapping $G^{c}$-orbits onto $G^{c}$-orbits and vectors of equal length into vectors of equal length.

Let $z \in C^{n}$ be a vector of minimum length, then its image $\zeta$ is a vector of minimum length (on its $G^{c}$-orbit in $R^{2 n}$ ). It is a straightforward calculation to check that the stationarity conditions at $z_{0}$ for the restriction to $\Omega=G^{c} \cdot z_{0}$ of the function $\|z\|^{2}$, can be written as a stationarity condition at $\gamma=1$ for the function $f_{z_{0}}(\gamma)$ $=\left\|\gamma \cdot z_{0}\right\|^{2}$, defined on $G^{c}$ :

$$
\left\langle z_{0}, t \cdot z_{0}\right\rangle=0, \quad \forall t \in \mathscr{G} .
$$

Therefore, using P6, the statement of Proposition 1 of [3] can be reformulated in the form proposed under the following items P12 and P13:

P12. Let $\zeta \in R^{2 n}$ be a vector of minimum length, then the euclidean vector space $R^{2 n}$ can be decomposed in the following sum of orthogonal subspaces:

$$
R^{2 n}=T_{\zeta} \oplus T_{\varepsilon \zeta} \oplus\{\zeta\} \oplus\{\varepsilon \zeta\} \oplus N_{\zeta}^{\text {res }} .
$$

In Eq. (3.9), the symbol $\{\zeta\}$ denotes the one-dimensional subspace of $R^{2 n}$ generated by the vector $\zeta$, the subspace $T_{\zeta} \oplus T_{\varepsilon \zeta}$ is formed by the tangent directions to the $G^{c}$-orbit at $\zeta$, while $N_{\zeta}$ res is defined by:

$$
N_{\zeta}^{c}=\{\zeta\} \oplus\{\varepsilon \zeta\} \oplus N_{\zeta}^{\text {res }} \text {. }
$$

The converse of $\mathbf{P 1 2}$ can only be proved for semisimple groups. 
$\mathbf{P 1 3}$ [3]. If $G$ is semisimple and if at $\zeta$ Eqs. (3.9) hold, then $\zeta$ is a vector of minimum length on $G^{c} \cdot \zeta$.

On the contrary, as shown in Example 5, the converse of $\mathbf{P 1 0}$ is false even in the assumption that $G$ is simple.

\section{Closed $G^{c}$-Orbits and Invariant Theory}

In this section we shall illustrate the connections between closed orbits and set of zeroes of invariant polynomial functions. We shall also show how convenient sets of invariant polynomial functions can be exploited to parametrize the closed orbits.

As shown in [16], the $G$-orbits in $R^{2 n}$ can be parametrized by means of a minimal integrity basis of the ring of polynomial invariant functions of $\zeta \in R^{2 n}$, a minimal integrity basis being a minimal set of $G$-invariant polynomial functions of $\zeta,\left\{\theta_{\alpha}(\zeta)_{1 \leqq \alpha \leqq q}\right.$, through which every $G$-invariant polynomial function of $\zeta, p(\zeta)$, can be written as a polynomial function $\hat{p}$ of the $\theta_{\alpha}$ 's:

$$
p(\zeta)=\hat{p}(\theta(\zeta)) .
$$

Every $G$-invariant function is clearly a constant on a $G$-orbit. Vice versa, given an integrity basis $\left\{\theta_{\alpha}\right\}_{1 \leqq \alpha \leqq q}$, the $G$-orbit through $\bar{\zeta} \in R^{2 n}$ can be defined as the real algebraic set formed by the solutions of the following equations:

$$
\theta_{\alpha}(\zeta)=\theta_{\alpha}(\bar{\zeta})
$$

Therefore, the map $\theta=\left(\theta_{1}, \ldots, \theta_{q}\right)$, mapping each $G$-orbit of $R^{2 n}$ into a point of $R^{q}$, is called "orbit map." The image of $R^{2 n}$ through the orbit map, $\theta\left(R^{2 n}\right)$, is a semialgebraic ${ }^{4}$ subset of $R^{q}$, which can be identified with the orbit space of the action of $G$ in $R^{2 n}$. In [15] (see also [5]) it has been shown how the defining algebraic equations and inequalities can be determined in a standard way.

Not all the results valid in the case of compact groups can be extended to complex reductive linear algebraic groups acting in $C^{n}$. Also in this case, for instance, Hilbert's theorem assures the existence of a finite integrity basis, $\left\{p_{a}(z)\right\}_{1 \leqq a \leq d}$, for the ring of polynomial invariant functions of $z \in C^{n}[8$, Theorem 5.9 , p. 160], but there is no $1: 1$ correspondence between the $G^{c}$-orbits and the image of $C^{n}$ in $C^{d}, p\left(C^{n}\right)$, through the orbit map $p(z)=\left(p_{1}(z), \ldots, p_{d}(z)\right)$.

This easily emerges in the examples we have just presented. In Example 1, for instance, the $G^{c}$-orbits cannot be distinguished by the elements of an integrity basis, since there is no $G^{c}$-invariant non-constant polynomial function of $z$. In Example 2, a minimal integrity basis is given by the unique function $p(z)$ $=z_{1}{ }^{2}+z_{2}{ }^{2}+z_{3}{ }^{2}$, which assumes different values on distinct closed orbits, but does not separate the closed orbit $\{z=0\}$ from the non-closed orbit formed by the isotropic vectors: on both orbits $p(z)=0$.

The reason why $G^{c}$-invariant polynomial functions cannot separate all the $G^{c}$ orbits can be easily understood: a polynomial function which is constant on a set $\mathscr{I}$, is necessarily constant on the smallest (closed) algebraic set containing $\mathscr{I}$. Thus,

\footnotetext{
${ }^{4}$ A semialgebraic set of $R^{2 n}$ is a subset defined by means of algebraic equations and inequalities
} 
polynomial $G^{c}$-invariant functions can at most separate $G^{c}$-stable (closed) algebraic subsets of $C^{n}$. In fact:

P14 [8, Corollary 1.2, p. 29]. An integrity basis separates the closed disjoint $G^{c}$ stable algebraic sets and consequently (see P2) the closed $G^{c}$-orbits.

Let now $p$ be a given orbit map and let $\mathscr{N}_{w}$ denote the algebraic set formed by the common zeros of the following polynomial equations in $z$ :

$$
p_{a}(z)-p_{a}(w)=0, \quad a=1, \ldots, d .
$$

Then, owing to P14, $\mathscr{N}_{w}$ does not depend on the particular choice of the orbit map $p$ and from our arguments it follows that:

P15. The closure of the $G^{c}$-orbit through $w$ is contained in the $G^{c}$-stable (closed) algebraic set $\mathscr{N}_{w}$.

As we shall see $\mathscr{N}_{w}$ does not generally coincide with $\overline{G^{c} \cdot w}$. However, from $\mathbf{P} 14$ and $\mathbf{P 5}$ one immediately realizes that:

P16. The sets $\mathscr{N}_{w}$ and $\overline{G^{c} \cdot w}$ contain a unique closed $G^{c}$-orbit, which is the lowestdimension $G^{c}$-orbit contained in $\mathscr{N}_{w}$.

The unicity of the closed orbit mentioned in P16 hints at the validity of the statement:

$\mathbf{P 1 7}[6]$. The symmetry of the unique closed $G^{c}$-orbit $\Omega_{0}$ contained in the closure of $a$ $G^{c}$-orbit $\Omega$ is maximal in the sense that, if $z \in \Omega$ and $w \in \Omega_{0}$ then $\left(G^{c}{ }_{z}\right) \cong\left(G^{c}{ }_{w}\right)$, i.e. $G^{c}{ }_{z}$ is conjugated in $G^{c}$ to a subgroup of $G^{c}{ }_{w}$ :

$$
G_{z}^{c} \cong \gamma G^{c}{ }_{w} \gamma^{-1}
$$

for at least a $\gamma \in G^{c}$.

Given a homogeneous integrity basis $\left(p_{1}, \ldots, p_{d}\right)$, the image in $C^{d}$ of the map:

$$
C^{n} \ni z \rightarrow\left(p_{1}(z), \ldots, p_{d}(z)\right) \equiv p(z) \in C^{d} .
$$

will be identified, by definition, with the orbit space of the action of $G^{c}$ in $C^{n}$. Note that distinct $G^{c}$-orbits of $C^{n}$ are not mapped into distinct points of the orbit space. In fact, the content of P14 and of its consequences P15 and P16 can be resumed, recalling P5, in the following statement:

P18. The points of the orbit space of the action of $G^{c}$ are in one-to-one correspondence with the closed $G^{c}$-orbits in $C^{n}$.

The orbit spaces of complex reductive linear algebraic groups are easier to determine than the orbit spaces of compact groups. Generally, the elements of an integrity basis are related by algebraic identities

$$
R_{a}\left(p_{1}(z), \ldots, p_{d}(z)\right)=0, \quad \forall z \in C^{n}, \quad 1 \leqq a \leqq k,
$$

where the $R_{a}$ 's are complex polynomial functions in $d$ variables. Standard methods suitable for the determination of these relations are known for large classes of linear groups in the mathematical literature [14]. 
It can be shown that:

P19 [5, Proposition 5.2, p. 55]. Given an orbit map p a faithful image of the orbit space can be got as the closed algebraic subset of $C^{d}$ formed by the common zeros of all the equations relating the elements of the integrity basis $\left(p_{1}, \ldots, p_{d}\right)$. In particular, if the basis is free, the orbit space can be identified with $C^{d}$.

The sets $\mathscr{N}_{w}$, defined in connection with the statement of $\mathbf{P} \mathbf{1 5}$, are clearly the inverse images by $p$ in $C^{n}$ of the points of the orbit space.

The connection between $G$-invariant polynomial functions and $G^{c}$-invariant ones is very simple to state.

Let $q(z)$ be a $G$-invariant polynomial function of $z$ :

$$
q(g \cdot z)=q(z), \quad \forall g \in G,
$$

and let us express $g \in G_{0}$ in terms of its Lie algebra elements: $g(u)=\exp \left(\sum_{1}^{r} u_{\alpha} t_{\alpha}\right)$,
$u \in R^{r}$.

Then Eq. (4.6), if $g$ is restricted to $G_{0}$, is equivalent to the following infinite set of relations:

$$
\left.\frac{\partial n}{\partial u_{\alpha 1} \ldots \partial u_{\alpha n}} q(g(u) \cdot z)\right|_{u=0}=0, \quad n=1,2, \ldots
$$

For $\gamma \in G_{0}{ }^{c}$ and $w \in C^{r}$, let us write: $\gamma(w)=\exp \sum_{1}^{r} w_{\alpha} t_{\alpha}$. Then Eqs. (4.7) imply:

$$
\left.\frac{\partial n}{\partial w_{\alpha 1} \ldots \partial w_{\alpha n}} q(\gamma(w) \cdot z)\right|_{w=0}=0, \quad n=1,2, \ldots,
$$

and therefore:

$$
q(\gamma \cdot z)=q(t), \quad \forall \gamma \in G_{0}{ }^{c},
$$

Therefore, recalling the definition of $G^{c}$ :

P20. Every G-invariant polynomial function of $z \in C^{n}$ is $G^{c}$ invariant.

The same is clearly not true of the $G$-invariant polynomial functions of $z$ and $z^{*}$, which are also required, in addition to the $G$-invariant polynomial functions of $z$, in order to separate the $G$-orbits.

\section{Spontaneous (Super)Symmetry Breaking in Globally Supersymmetric Theories}

\section{Introduction}

As well known [1], in globally supersymmetric theories with compact connected ${ }^{5}$ internal symmetry group $G$ the spontaneous breaking of supersymmetry and/or internal symmetry is controlled by the minima of an effective potential

\footnotetext{
${ }^{5}$ This assumption is not essential for the validity of all our subsequent conclusions
} 
$V\left(z, z^{*}\right)$, which depends only the scalar fields $z=\left(z_{1}, \ldots, z_{n}\right)$ of the chiral supermultiplets through the gradient of the superpotential $f(z)$ (a $G$-covariant polynomial function of $z$ ) and additional geometric terms, when the internal symmetry is local. Owing to translation invariance of the vacuum in Minkowsky space, in the search of the minima of $V$, it is not too restrictive to consider $z$ as a vector (or a point) of $C^{n}$. The group $G$ will be considered in the following as a group of linear unitary transformations of $C^{n}$.

As stressed in the general Introduction the complexification $G^{c}$ of the group $G$, plays an important role in the search and characterization of the points where $V\left(z, z^{*}\right)$ takes on its minimum and is relevant in the determination of those degeneracies of the vacuum state that are related to the $G$-symmetry; these will be called $G$-symmetry-related (abbreviated into GSR) degeneracies of the vacuum.

In the second part of the paper we shall show how the mathematical results presented in Part I can be translated into precious information in this context. Theories with global and local internal symmetry will be discussed separately, on the tacit assumption, when necessary, that $V\left(z, z^{*}\right)$ takes on an absolute minimum (at a finite point).

General properties will be pointed out both of the residual internal symmetry groups after spontaneous symmetry breaking and of the group of transformations relating the GSR degenerate vacua. Like all continuous degeneracies of the vacuum state, the GSR ones will be responsible for the existence of a certain number of massless particles; the constraints on the mass spectrum of theories with possibly spontaneously broken global supersymmetry, implied by these degeneracies, will be derived in a subsequent paper [17].

We shall subsequently report the details of a recent geometrical and analytical characterization of the zeroes of the $D$-term $[3,6]$. This will allow us to propose a correct proof of a theorem stated in Bagger and Wess [1], concerning necessary and sufficient conditions for spontaneous breaking of supersymmetry. We shall also show that the $D$-term necessarily vanishes at every point where the effective potential takes on its minimum if the gradient of the superpotential is invariant by the internal symmetry group at a point where the $F$-term takes on its minimum. Some consequences of the assumption that the vacuum corresponds to a stationary point of the $F$-term will finally be worked out.

In the following $\partial_{i}$ and $\partial_{i}{ }^{*}$ will denote differentiation with respect to $z_{i}$ and $z_{i}{ }^{*}$, respectively, but we shall omit indices when this will not give rise to ambiguities.

\section{Globally Supersymmetric Theories with Global Internal Symmetry}

In globally supersymmetric theories with global internal symmetry the effective potential has the following form:

$$
V\left(z^{\prime}, z^{\prime *}\right)=F\left(z^{\prime}, z^{\prime *}\right)=\left\|\partial f\left(z^{\prime}\right)\right\|^{2},
$$

where $\partial f\left(z^{\prime}\right)$ denotes the gradient at $z^{\prime}$ of the superpotential $f\left(z^{\prime}\right)$ and is a $G$ covariant polynomial function of $z^{\prime}$. In this section we shall derive the consequences of this particular structure of the potential or, more exactly, of the fact that, according to $\mathbf{P 2 0}$, the gradient of the superpotential is $G^{c}$-covariant. 
As well known, the action of $G^{c}$ in $C^{n}$

$$
\left(\gamma \cdot z^{\prime}\right)_{i}=\sum_{1}^{n} \gamma_{i j} z^{\prime}
$$

induces on the space of gradients of analytical $G^{c}$-invariant functions of $z^{\prime}$ a dual action of $G^{c}$ :

$$
\partial_{i} f\left(\gamma \cdot z^{\prime}\right)=\sum_{1}^{n}\left(\gamma^{T-1}\right)_{i j} \partial_{j} f\left(z^{\prime}\right)
$$

where $T$ denotes transposition.

The function $\partial f\left(z^{\prime}\right)$ maps clearly the whole of $V \simeq C^{n}$ onto a $G^{c}$-invariant subset of $V \dagger \simeq C^{n}$, and the linear group $G^{c} \dagger=\left\{\gamma \dagger \mid \gamma \dagger=\gamma^{T-1}, \gamma \in G^{c}\right\}$ yields a rational representation of $G^{c}$ and is therefore a linear reductive group. Both for the action of $G^{c}$ on the vectors of $V$ and $V \dagger$ we shall use the same notation, and similarly for the action of $\mathscr{G}^{c}$ in $V$ and $V \dagger$. So, for instance, Eq. (2.2b) will be written synthetically:

$$
f\left(\gamma \cdot z^{\prime}\right)=\gamma \cdot f\left(z^{\prime}\right)
$$

From Eq. (2.3) one immediately realizes that:

$$
G_{z}^{c} \subseteq G_{\partial f(z)}^{c}, \quad \forall z \in C^{n},
$$

and consequently:

$$
\mathscr{G}_{z}{ }^{c} \subseteq \mathscr{G}^{c}{ }_{\partial f(z)}, \quad \forall z \in C^{n} .
$$

So, when $V\left(z^{\prime}, z^{*}\right)$ takes on its minimum at $z$, the complex symmetry of the direction $z$ of spontaneous internal symmetry breaking cannot exceed the complex symmetry of the direction $\partial f(z)$ of spontaneous supersymmetry breaking.

Below, after deriving some general properties of the groups $G_{\partial f(z)}^{c}$ and $G_{z}^{c}$ we shall discuss the consequences of Eq. (2.5), when the inclusion is satisfied in a strict sense. This is likely to occur, for instance, if $\partial f\left(z^{\prime}\right)=0$ has solutions $z \neq 0$, or when $F$ takes on its minimum in correspondence of non-vanishing values of all noninvariant components of $z$.

Proposition 2.1. Let $\Omega=G^{c} \cdot z$. If $z$ is a stationary point of $\left.\left\|\partial f\left(z^{\prime}\right)\right\|^{2}\right|_{\Omega}$, then it corresponds to an absolute minimum and the isotropy subgroups of the dual action of $G^{c}$ at $\varphi=\partial f(z)$, satisfies the following condition:

$$
G_{\varphi}^{c}=G_{\varphi}^{c},
$$

so that it is, in particular, reductive.

Proof. In the assumptions of Proposition 3.1, the function $h_{\varphi}: G^{c} \rightarrow R^{1}{ }_{+}$, defined by $h_{\varphi}(\gamma)=\|\partial f(\gamma \cdot z)\|^{2}=\|\gamma \cdot \varphi\|^{2}$, has a stationary point at $\gamma=1$. From P6, P7, and P9 we learn that $\|\varphi\|^{2}$ is the minimum of $h_{\varphi}(\gamma)$, the orbit $G^{c} \cdot z$ is closed and Eq. (2.6) is satisfied.

Remark 1. When supersymmetry is not spontaneously broken, $\partial f(z)=0$, and Eq. (2.6) reduces to a well known result [2]: $G_{\varphi=0}^{c}=G_{\varphi=0}{ }^{c}=G^{c}$.

Remark 2. If $z$ is a stationary point of $F\left(z^{\prime}, z^{*}\right)$, it is, in particular, a stationary point of $\left.F\left(z^{\prime}, z^{*}\right)\right|_{\Omega}$, where $\Omega=G^{c} \cdot z$; therefore, the conclusions of Proposition 2.1 hold at $z$. 
Using $\mathbf{P 8}$ one can immediately identify all those degeneracies of the minimum of the effective potential which are a consequence of the $G^{c}$-invariance of the superpotential:

Proposition 2.2. Let $V\left(z^{\prime}, z^{*}\right)$ take on its minimum at $z$ and let $\varphi=\partial f(z)$. Then, except for the points lying on the set $\Sigma_{z}=G \cdot G_{\varphi}^{c} \cdot z$, there are no other points on the orbit $G^{c} \cdot z$, where the $V\left(z^{\prime}, z^{*}\right)$ takes on its minimum.

The following Example 6 will illustrate the content of Propositions 2.1 and 2.2

Example 6. Let $G$ be a $\underline{2}+\underline{3}$ unitary matrix representation of $S U_{2}$, analogous to the representation of $\mathrm{SU}_{3}$ defined in Example 5, and let us choose as superpotential the function,

$$
f=u \lambda^{2}+w^{T} i \sigma_{2} \lambda w+a u+b v
$$

where the $\sigma_{j}$ 's are Pauli's matrices, $a$ and $b$ are complex constants, $u$ and $v$ are complex singlets of $G$ and $\left(\lambda_{1}, \lambda_{2}, \lambda_{3}\right) \equiv \lambda$ are the complex components of the matrix $\lambda$ in the basis $\left\{\sigma_{j}\right\}_{j=1,2,3}$ :

$$
\lambda=\sum_{1}^{3} \lambda_{j} \sigma_{j}=\lambda \cdot \sigma
$$

From Eq. (2.7) we obtain:

$$
f=\left(\begin{array}{c}
2 i \sigma_{2} \lambda w \\
2 u \lambda+w^{T} i \sigma_{2} \sigma w \\
\lambda^{2}+a \\
b
\end{array}\right) .
$$

It is not difficult to realize that the function $\left\|\partial f\left(z^{\prime}\right)\right\|^{2}$ takes on its minimum at the points $z$ characterized by the following relations:

$$
w=0=u ; \quad \lambda^{2}=-a .
$$

The vector $\varphi=\partial f(z)$ turns out to be invariant by $G^{c}=S L_{2}(C)$ and its squared norm-equals $|b|^{2}>0$. There is, therefore, a surface of degenerate minima $S$, whose equation is (2.10). Let us call $S_{0}$ the intersection of $S$ with the hyperplane of equation $v=v_{0}$ and discuss the two cases $a=0$ and $a \neq 0$ separately.

For $a \neq 0$, the surface $S_{0}$ coincides with a unique closed $G^{c}$-orbit whose complex symmetry is $\left(G L_{1}(C)\right)$.

As for the compact symmetry of points of $S_{0}$, for $a \neq 0$, there are the following two possibilities i) and ii):

i) for general $z$ in $S_{0}$ one gets $G_{z}=\{ \pm 1\}$, so that $G_{z}^{c} \neq G_{z}{ }^{c}$;

ii) for $z \propto \xi$, where $\xi$ is a real vector, one gets $G_{z} \simeq U_{1}$, so that, in this case, $G^{c}{ }_{z}=G_{z}^{c}$.

For $a=0$ the surface $S_{0}$ is the union of a non-closed $\left(\lambda^{2}=0 \neq \lambda\right)$ and a closed $(\lambda=0)$ orbit.

At the points $z$ of the non-closed orbit $G_{z}=\{ \pm 1\}$, while $G_{z}^{c}$ is isomorphic to the additive group of the complex numbers, a non-reductive algebraic group, multiplied by $Z_{2}=\{ \pm 1\}$. 
The following Example 7 yields a sample of a situation in which supersymmetry is broken in a direction which is not invariant by $G, G_{\varphi}^{c} \neq G^{c}$, but $G_{\varphi}^{c}$ contains $G^{c}{ }_{z}$ in a strict sense.

Example 7. Let us consider a representation $3 \cdot\left(3^{c}+1^{c}\right)$ of $S O_{3}(R)$. Then $z^{\prime}$ is the direct sum of three complex 3-dimensional vectors $\mathbf{z}_{i}$ and three complex singlets $s_{i}$, $i=1,2,3$, and $G^{c} \simeq S O_{3}(C)$. Let us choose as superpotential the invariant function $f:$

$$
f=k\left(\mathbf{z}_{1} \times \mathbf{z}_{2}\right) \cdot \mathbf{z}_{3}+s_{1}\left(\mathbf{z}_{1}{ }^{2}-1\right)+s_{2}\left(\mathbf{z}_{2}{ }^{2}-1\right)+k^{\prime} s_{3} \mathbf{z}_{1} \cdot \mathbf{z}_{2},
$$

where $k$ and $k^{\prime}$ are complex constants and

$$
0<|k|<\left|k^{\prime}\right| \text {. }
$$

From Eqs. (2.11):

$$
f=\left(\begin{array}{c}
k \mathbf{z}_{2} \times \mathbf{z}_{3}+2 s_{1} \mathbf{z}_{1}+k^{\prime} s_{3} \mathbf{z}_{2} \\
k \mathbf{z}_{3} \times \mathbf{z}_{1}+2 s_{2} \mathbf{z}_{2}+k^{\prime} s_{3} \mathbf{z}_{1} \\
k \mathbf{z}_{1} \times \mathbf{z}_{2} \\
\mathbf{z}_{1}{ }^{2}-1 \\
\mathbf{z}_{2}{ }^{2}-1 \\
k^{\prime} \mathbf{z}_{1} \cdot \mathbf{z}_{2}
\end{array}\right) .
$$

It is easy to realize that $\|\partial f\|$ cannot vanish and, attains its minimum, for instance, for:

$$
s_{i}=0=\mathbf{z}_{3}, \quad i=1,2,3 .
$$

In fact, with this choice, the first two vector components of $\partial f$ vanish, while the remaining ones are completely unconstrained. Therefore, the minimum of $F$ coincides with the minimum of the function $F_{0}$ defined by:

$$
\begin{gathered}
F_{0}=\left.F\right|_{s_{2}=0=\mathbf{z}_{3}=|k|^{2}\left(\left\|\mathbf{z}_{1}\right\|^{2}\left\|\mathbf{z}_{2}\right\|^{2}-\left|\mathbf{z}_{1} \cdot \mathbf{z}_{2}\right|^{2}\right)} \\
+\left|\mathbf{z}_{1}{ }^{2}-1\right|+\left|\mathbf{z}_{2}{ }^{2}-1\right|+\left|k^{\prime}\right|^{2}\left|\mathbf{z}_{1} \cdot \mathbf{z}_{2}\right|, \\
\frac{\partial F_{0}}{\partial \mathbf{z}_{1}{ }^{*}}=|k|^{2}\left(\left\|\mathbf{z}_{2}\right\|^{2} \mathbf{z}_{1}-\left(\mathbf{z}_{1} \cdot \mathbf{z}_{2}{ }^{*}\right) \mathbf{z}_{2}\right)+2\left(\mathbf{z}_{1}{ }^{2}-1\right) \mathbf{z}_{1}{ }^{*}+\left|k^{\prime}\right|^{2}\left(\mathbf{z}_{1} \cdot \mathbf{z}_{2}\right) \mathbf{z}_{2}{ }^{*}=0 \\
\frac{\partial F_{0}}{\partial \mathbf{z}_{2}{ }^{*}}=|k|^{2}\left(\left\|\mathbf{z}_{1}\right\|^{2} \mathbf{z}_{2}-\left(\mathbf{z}_{1}{ }^{*} \cdot \mathbf{z}_{2}\right) \mathbf{z}_{1}\right)+2\left(\mathbf{z}_{2}{ }^{2}-1\right) \mathbf{z}_{2}{ }^{*}+\left|k^{\prime}\right|^{2}\left(\mathbf{z}_{1} \cdot \mathbf{z}_{2}\right) \mathbf{z}_{1}{ }^{*}=0
\end{gathered}
$$

After some lengthy but straightforward calculations one finds the following exhaustive set of solutions, i)-iv), of the stationarity conditions, Eqs. (2.15); under the same items are also indicated the corresponding values of $F_{0}$ :

i) $\mathbf{z}_{1}=\mathbf{z}_{2}=0 ; F_{0}=2$;

ii) $\mathbf{z}_{1}=0, \mathbf{z}_{2}{ }^{2}=1$, or $\mathbf{z}_{2}=0, \mathbf{z}_{1}{ }^{2}=1 ; F_{0}=1$;

iii) $\mathbf{z}_{2}= \pm \mathbf{z}_{1} \neq 0$;

iv) $\mathbf{z}_{1}=h \boldsymbol{\xi}+i h^{\prime} \boldsymbol{\xi}^{\prime}, \mathbf{z}_{2}= \pm\left(h \xi^{\prime}-i h^{\prime} \boldsymbol{\xi}\right)$, where $\boldsymbol{\xi}$ and $\xi^{\prime}$ are mutually orthogonal arbitrary unit real vectors and $h, h^{\prime}$ are real numbers satisfying only the following 
conditions:

$$
\begin{gathered}
h>h^{\prime} \geqq 0, \\
h^{2}-h^{\prime 2}=\left(1+k^{2} / 2\right)^{-1} ;
\end{gathered}
$$

moreover,

$$
F_{0}=2 \frac{|k|^{2}}{2+|k|^{2}} .
$$

Owing to Eq. (2.11b), $F$ takes on its absolute minimum, which coincides with the right-hand side of Eq. (2.17), at the stationary points of $F$ which are associated, as we have seen, to the stationary points of $F_{0}$ defined under item iv). At the points of the surface $\sum_{z}$ of degenerate minima of $F$ we have found:

$$
\varphi=\partial f=\left(\begin{array}{c}
0 \\
0 \\
\pm k K \xi \times \xi^{\prime} \\
K-1 \\
K-1 \\
0
\end{array}\right)
$$

where

$$
K=\frac{1}{1+k^{2} / 2} .
$$

Therefore, $G_{z}=\{1\}=G_{z}^{c}=G_{z}^{c}, G_{\varphi}\left(G_{\varphi}^{c}\right)$ is the subgroup of $\mathrm{SO}_{3}(R)\left(\mathrm{SO}_{3}(C)\right)$ formed by the complex proper rotations around the axis $\xi \times \xi^{\prime}$, so that $G_{\varphi}^{c}=G_{\varphi}{ }^{c}$, in agreement with Proposition 2.1, and $G_{\varphi}^{c} \supset G_{z}^{c}$ in a strict sense. The surface $\Sigma_{z}$ is easily seen to coincide with the set $G \cdot G_{\varphi}^{c} \cdot z$. In fact, let us choose $\xi$ and $\xi^{\prime}$, respectively, in the directions of the $x$ and $y$ axes and $h, h^{\prime}$ in their allowed ranges. Then it is easy to check that any other allowed choice of $h$ and $h^{\prime}$ with the same $\xi$ and $\xi^{\prime}$, corresponds to a rotation $\gamma$ of $G^{c}{ }_{\varphi}$ with imaginary angle ia:

$$
\gamma=\left(\begin{array}{ccc}
\cos i a, & -\sin i a, & 0 \\
\sin i a, & \cos i a, & 0 \\
0, & 0, & 1
\end{array}\right),
$$

while any other choices of $\xi$ and $\xi^{\prime}$ with the same values of $h$ and $h^{\prime}$, can be obtained by means of a real rotation induced by $G$. In order to determine all the points where $F$ takes on its minimum, one has to require the vanishing of the first two components of $\partial f(z)$, in correspondence with the values of $\mathbf{z}_{1}$ and $\mathbf{z}_{2}$ specified under item iv). After some straightforward calculations one finds

$$
\mathbf{z}_{3}=-\frac{2 s}{k} \xi \times \xi^{\prime}, \quad s_{1}=s_{2}=s, \quad s_{3}=0
$$

where $s$ is an arbitrary complex constant. Therefore, also in this case, as expected [18], the degeneracy of the minimum on $\Sigma_{z}$, due to the complex character of the 
internal symmetry, is superimposed on an additional degeneracy along a linear subspace whose equations are given by Eqs. (2.20) and

$$
\mathbf{z}_{1}=h \xi+i h^{\prime} \xi^{\prime}=\text { const }, \quad \mathbf{z}_{2}= \pm\left(h \xi^{\prime}-i h^{\prime} \xi\right) .
$$

As a consequence the potential is not confining [20].

Contrary to what happens for $G^{c}$, the isotropy subgroup of $G^{c}$ at $z$ need not satisfy Eq. (2.6), nor be reductive; however, as we shall prove below, there is always a degenerate minimum of the potential at a point $\bar{z}$ where the isotropy subgroup is reductive and satisfies Eq. (2.6).

Proposition 2.3. Let $z$ be a point where the effective potential takes on its minimum, then there exists a point $\bar{z}$ where:

i) $V\left(\bar{z}, \bar{z}^{*}\right)=V\left(z, z^{*}\right)$ :

ii) $G_{\bar{z}}^{c}=G_{\bar{z}}{ }^{c}$; and

iii) $G_{z}$ is conjugated in $G^{c}$ to a subgroup of $G_{\bar{z}}$.

Proof. Let us define $\varphi=\partial f(z)$ and consider the closure $\bar{\Omega}$ of the $G^{c}{ }_{\varphi}$-orbit $\Omega$ through $z$. By continuity $\partial f(z)=\varphi$ on $\bar{\Omega}$. Moreover, from Proposition 2.1 we know that $G_{\varphi}^{c}$ is reductive, so we can use $\mathbf{P} \mathbf{1 6}$ (where $G_{\varphi}^{c}$ has to be substituted for $G^{c}$ ), to claim that in the set $\bar{\Omega}$ there is a unique closed $G^{c}{ }_{\varphi}$-orbit $\Omega^{\prime}$. Owing to Eq. (2.4), the isotropy subgroups of the two groups $G^{c}$ and $G^{c}{ }_{\varphi}$ are identical at points of $\Omega^{\prime}$. So, from P17 we deduce the following constraints for the isotropy subgroup of $G^{c}$ at every $z^{\prime} \in \Omega^{\prime}:\left(G_{\varphi}^{c}\right)_{z^{\prime}}=G_{z^{\prime}}^{c} \supseteqq \gamma G_{z}^{c} \gamma^{-1}$. In particular, owing to $\mathbf{P 9}$, the isotropy subgroups of the vectors $\bar{z}$ of minimum length on $\Omega^{\prime}$ will satisfy $G_{\bar{z}}^{c}=G_{\bar{z}}{ }^{c}$. Therefore, using Eq. (I.1.9) we obtain for a convenient $\gamma \in G^{c}$ : $G_{\bar{z}}{ }^{c}=G_{\bar{z}}^{c} \supseteqq \gamma G_{z}^{c} \gamma^{-1} \supseteqq \gamma G_{z}{ }^{c} \gamma^{-1}$.

Now, $\gamma G_{z} \gamma^{-1}$ is a compact subgroup of $\gamma G_{z}{ }^{c} \gamma^{-1}$, which is contained in $G_{\bar{z}}{ }^{c}$, as we have seen; therefore, $\gamma G_{z} \gamma^{-1}$ is conjugated in $G^{c}$ to a subgroup of $G_{\bar{z}}$, which is a maximal compact subgroup of $G_{\bar{z}}{ }^{c}$. This achieves the proof of Proposition 2.3.

Remark 3. In the proof of Proposition 2.3, we have also shown that at all the points of the set $G \cdot \overline{\left(G_{\varphi}^{c} \cdot z\right)}=\overline{G \cdot\left(G_{\varphi}^{c} \cdot z\right)}=\bar{\Sigma}_{z}$, the function $F$ takes on its minimum. These degenerate minima are a direct consequence of the $G^{c}$-invariance of the superpotential. Obviously, there may be other degeneracies depending on the particular values of the parameters determining the superpotential and/or on the upper bound on its degree.

It is not difficult to calculate the real dimension of $\Sigma_{z}$ at $z$. One finds:

$$
\operatorname{dim} \Sigma_{z}=\operatorname{dim}_{c}\left(\mathscr{G}^{c}-\mathscr{G}_{z}^{c}\right)+\operatorname{dim}_{c}\left(\mathscr{G}_{\varphi}^{c}-\mathscr{G}_{z}^{c}\right) .
$$

The dimension $\Sigma_{z}$ at $z$ sets a lower limit to the number of massless scalar particles of the theory in tree approximation.

Propositions 2.1-2.3 can be used to claim the existence of continuously degenerated vacua in all the cases in which a non-reductive residual symmetry [11] has been advocated in globally supersymmetric models. This has been the case, for instance in supersymmetric preonic models [10].

It is interesting to compare theories based on different degenerate vacua, when $\mathscr{G}_{\varphi}^{c}$ contains strictly $\mathscr{G}_{z}^{c}$, i.e. when the complex symmetry of the direction of supersymmetry breaking is larger than the symmetry of the direction of internal symmetry breaking. 
Let $\bar{z}$ be a point where $V\left(z^{\prime}, z^{\prime *}\right)$ takes on its minimum, then the same occurs at all the points of the set $\Sigma_{\bar{z}}=G \cdot G_{\bar{\varphi}}^{c} \cdot \bar{z}, \varphi=\partial f(z)$. By varying $z$ on $\Sigma_{\bar{z}}$, the residual (compact) symmetry after spontaneous symmetry breaking, $\left(G_{z}\right)$, and therefore, the number of Goldstone particles, may be generally changed, since $G_{g \cdot z}$ is in general $\neq g G_{z} g^{-1}$, for $g \in\left(G^{c}-G\right)$.

On the contrary, the complex symmetry $\left(G_{z}^{c}\right)$, as well as $\operatorname{dim} \Sigma_{z}$ at $z$, remain the same.

If the following subset $S$ of degenerate minima: $S=\left\{z \in C^{n} \mid \partial f(z)=\bar{\varphi}\right\}$ does not reduce to a unique $G_{\bar{\varphi}}^{c}$-orbit ${ }^{6}$ (which certainly occurs, for instance if $G_{\bar{\varphi}}^{c} \cdot \bar{z}$ is not closed), by shifting $z$ through different $G^{c}{ }_{\varphi}$-orbits, the complex residual symmetry may be changed, too. In particular, the class $\left(G_{z}^{c}\right)$ will turn out to be locally maximal on closed $G_{\varphi}^{c}$-orbits, which certainly exist inside $S$. Note also that, on a closed $G^{c}$-orbit, the class $\left(G_{z}\right)$ is maximum when $z$ is a vector of minimum length and, in this case, $G_{z}$ is a maximal compact subgroup of $G_{z}^{c}$.

As we shall see in the next section, the $G^{c}$-related degeneracy of the vacuum is completely removed when the group $G$ is completely gauged. Therefore, in that case one cannot exploit the possibility of varying $z$ on $\bar{\Sigma}_{\bar{z}}$ in order to vary the number of massless chiral particles (after the Higgs mechanism has taken place) in theories with conserved supersymmetry and spontaneously broken gauge symmetry.

\section{Globally Supersymmetric Gauge Theories}

In this section we shall be concerned with globally supersymmetric theories with local internal symmetry and no Fayet-Iliopoulos term [19]. As well known in these theories the effective potential is the sum of two non-negative terms:

$$
V\left(z^{\prime}, z^{*}\right)=F\left(z^{\prime}, z^{*}\right)+D\left(z^{\prime}, z^{*}\right)=\left\|\partial f\left(z^{\prime}\right)\right\|^{2}+\frac{1}{2} \Sigma_{\alpha} g_{\alpha}{ }^{2}\left|D_{\alpha}\left(z^{\prime}, z^{\prime *}\right)\right|^{2},
$$

where the components $D_{\alpha}\left(z^{\prime}, z^{\prime}\right)$ of the $D$-term are defined by:

$$
D_{\alpha}\left(z^{\prime}, z^{*}\right)=\left(z^{\prime}, t_{\alpha} z^{\prime}\right)=-D_{\alpha}\left(z^{\prime}, z^{*}\right)^{*},
$$

the $g_{\alpha}$ 's are gauge coupling constant and $\left\{t_{\alpha}\right\}_{1 \leqq \alpha \leqq r}$ is an orthonormal basis in $\mathscr{G}$ [see Eq. (I.2.2)].

As is well known, supersymmetry is not spontaneously broken if and only if the absolute minimum of the effective potential is zero.

It is, therefore, important to determine all the zeroes of the $D$-term. As argued in [3] (see Remark d) and [5] there is a strict connection between zeroes of the $D$ term and closed $G^{c}$-orbits. Below we shall make this connection precise using the results of Part $\mathrm{I}$.

The link rests on the following remark. Let us set $\Omega=G^{c} \cdot z$, then the stationarity conditions for the function $\left.\left\|z^{\prime}\right\|^{2}\right|_{\Omega}$ at $z \in \Omega$ reduce to Eqs. (I.3.8), that is $D\left(z, z^{*}\right)=0$.

The zeroes of the $D$-term are characterized by the following Proposition 3.1 $[3,6]$.

\footnotetext{
${ }^{6}$ Note that $S$ contains at least one point of every $G$-orbit lying in $\bar{\Sigma}_{\bar{z}}$
} 
Proposition 3.1. If the D-term annihilates at $z$ then the $G^{c}$-orbit through $z$ is closed. Vice versa, the D-term annihilates on every closed $G^{c}$-orbit $\Omega$ at points lying on a unique $G$-orbit formed by all the vectors of minimum length on $\Omega$. Moreover, at every zero of the D-term, $G_{z}{ }^{c}=G_{z}^{c}$.

Proof. The statement is an immediate consequence of Eq. (I.3.8) and P6, P7, P8, P9.

Let us consider the set of $G$-orbits formed by zeroes of the $D$-term. From Proposition 3.1, its element turn out to be in one-to-one correspondence with the closed $G^{c}$-orbits and therefore with the points of the orbit-space of the $G^{c}$-action in $C^{n}$ : the orbit space of $G^{c}$ yields a faithful image of the set of $G$-orbits formed by zeroes of the $D$-term. This image can be explicitly determined according to P19.

There is an alternative way of characterizing the zeroes of the $D$-term, based on an Ansatz proposed by Buccella et al. [20].

Proposition 3.2. The D-term annihilates at a point $z$ if and only if there is a polynomial invariant function of $z^{\prime}, h\left(z^{\prime}\right)$, such that:

$$
z^{*}=\partial h(z) \text {. }
$$

Proofs can be found in $[5,3]$. For completeness we report here the line of the proof.

Proof. The sufficiency of Eq. (3.2) is an immediate consequence of the covariance of the gradient of $h$, which yields: $\left((\partial f(z))^{*}, \tau \cdot z\right)=0, \forall z \in C^{n}$ and $\tau \in \mathscr{G}^{c}$.

The necessity is also simple to prove in a large class of situations. Let us assume that $z$ is a zero of the $D$-term, call $\left\{p_{\alpha}(z)\right\}_{1 \leqq \alpha \leqq d}$ a minimal integrity basis and denote by $\mathscr{N}_{z}$ the closed algebraic set of the solutions $z^{\prime}$ of the following equations:

$$
p_{\alpha}\left(z^{\prime}\right)-p_{\alpha}(z)=0, \quad \alpha=1, \ldots, q .
$$

Since $\mathscr{N}_{z}$ is closed, there are points on it at minimum distance from the origin, and using P16, P7, and P8 it is not difficult to realize that they form a unique $G$ orbit inside the unique closed $G^{c}$-orbit contained in $\mathscr{N}_{z}$. The points on $\mathscr{N}_{z}$ at minimum distance from the origin are stationary points of the function $n\left(z^{\prime}, z^{*}\right)$ $=\left.\left\|z^{\prime}\right\|^{2}\right|_{\mathscr{N}_{z}}$. The condition for a (constrained) stationary point of $n\left(z^{\prime}, z^{\prime *}\right)$ at $z$ can be written by means of $q$ Lagrange multipliers $\lambda_{a}$ as:

$$
\partial\|z\|^{2}=z^{*}=\sum_{1}^{d} \partial p_{a}(z) \lambda_{a}
$$

which, for $h(z)=\sum_{1}^{d} p_{a}(z) \lambda_{a}$, coincides with Eq. (3.2).

The simple proof of the necessity of the condition expressed in Eq. (3.2) for the vanishing of the $D$-term, given above, is not complete. In fact, the Lagrange multipliers method is not safe when the matrix formed with the gradients of the constraint functions $p_{\alpha}(z)$ has not constant rank on $\mathscr{N}_{z}$ [3]. This may be the case if $\mathscr{N}_{z}$ does not reduce to a set of $G^{c}$-orbits with the same complex symmetry.

A complete proof of the necessity of Eq. (3.2) requires the use of a much more sophisticated mathematics $[5,21]$ and can be achieved by refining the arguments that led us to prove, in [3], a slightly weaker result: if at $z, D\left(z, z^{*}\right)$ vanishes, then 
there exists a real polynomial function of $z$ and $z^{*}, I\left(z, z^{*}\right)$, such that:

$$
i z^{*}=\partial I\left(z, z^{*}\right) \text {. }
$$

Let us recall the proof of this fact. Let $z$ be a zero of $D\left(z, z^{*}\right)$ and $\zeta$ the real image of $z$ in $R^{2 n}$. Then from $\mathbf{P} 12$ we know that $\varepsilon \zeta \in N^{c}{ }_{\zeta} \subset N_{\zeta}$, or more exactly, $\varepsilon \zeta$ belongs to the subspace $N^{c, 0}{ }_{\zeta}$ of $N_{\zeta}^{c}$ formed by the vectors of $N^{c}$ which are invariant by $G_{z}^{c}$. Therefore, since the subspace $N^{0}{ }_{\zeta}$, of $N_{\zeta}$, formed by the $G_{z}$-invariant vectors is spanned by the gradients at $\zeta$ of the elements $\theta_{\alpha}(\zeta), 1 \leqq \alpha \leqq q_{1},[22]$ of a minimal integrity basis for the ring of $G$-invariant real polynomial functions of $\zeta$, the vector $\varepsilon \zeta$ can be written in the following way:

$$
\varepsilon \zeta=\sum_{1}^{q_{1}} a_{\alpha} \partial \theta_{\alpha}(\zeta)
$$

where $a_{\alpha} \in R^{1}$. It is easy to check that Eq. (3.6) is equivalent to Eq. (3.5). In order to derive Eq. (3.2) from Eq. (3.6) we need some further information. To this end let us note that the $\theta_{\alpha}$ 's can be thought of as originating from real $G$-invariant polynomial functions of $z$ and $z^{*}$, and can be chosen so that, for $1 \leqq \alpha \leqq q, p_{\alpha}(z)$ $=\theta_{q+\alpha}(\zeta)+i \theta_{\alpha}(\zeta)$, where $\left\{p_{\alpha}(z)\right\}_{1 \leqq \alpha \leqq q}$ is a minimal integrity basis for the ring of $G^{c}$ invariant polynomial functions of $z$. The holomorphism conditions for $p_{\alpha}(z)$ can be expressed in the following form:

$$
\varepsilon \partial \theta_{n+\alpha}(\zeta)=\partial \theta_{\alpha}(\zeta), \quad \alpha=1, \ldots, q .
$$

It has been shown in [6] that, as a consequence of Luna's slice theorem [15], the subspace $N^{c, 0}{ }_{\zeta}$ is in fact generated by the gradients of the invariants $\theta_{\alpha}(\zeta)$, $1 \leqq \alpha \leqq 2 q$, originating from the elements of the integrity basis $\left\{p_{\alpha}(z)\right\}_{1 \leqq \alpha \leqq q}$.

Therefore, the sum in Eq. (3.6) can be arrested at $\alpha=2 q<q_{1}$, so that, using also (3.7) and the fact that $\varepsilon^{2}=-1$ Eq. (3.6) can be rewritten in the following form:

$$
\zeta=\sum_{1}^{q}\left(a_{\alpha} \varepsilon+a_{n+\alpha}\right) \partial \theta_{\alpha}(\zeta)
$$

From Eq. (3.8) we immediately derive:

$$
\begin{aligned}
i z^{*} & =-i \sum_{1}^{q}\left(a_{\alpha}+i a_{n+\alpha}\right)\left(\partial_{j}-i \partial_{n+j}\right) \theta_{\alpha}(\zeta) \\
& =\sum_{1}^{q} c_{\alpha} \partial p_{\alpha}(z) / \partial z_{j}=\frac{\partial p(z)}{\partial z_{j}}
\end{aligned}
$$

where $c_{\alpha}=-a_{\alpha}-i a_{n+\alpha}, \alpha=1, \ldots, q$ and $p(z)=\sum_{1}^{q} c_{\alpha} p_{\alpha}(z)$.

It is commonly accepted that the possibility of spontaneously breaking the supersymmetry depends on the existence of zeroes of the $F$-term. This claim, which we shall formalize in the following Proposition $3.3^{7}$, is correctly stated, but not proved, by Bagger and Wess [1].

Proposition 3.3. The minimum of the potential vanishes if and only if the F-term vanishes somewhere.

\footnotetext{
${ }^{7}$ Some of the arguments of [4] can be used to prove Proposition 3.3 (see, however, footnote 2)
} 
Proof. The only non-trivial part of the statement is the claim that the condition

$$
\min _{z^{\prime} \in C^{n}} F\left(z^{\prime}, z^{\prime *}\right)=0
$$

implies:

$$
\min _{z^{\prime} \in C^{n}} V\left(z^{\prime}, z^{*}\right)=0
$$

Let us assume that Eq. (3.10) is satisfied and call $\mathscr{N}$ the set of zeroes of $\partial f\left(z^{\prime}\right)$. Then $\mathscr{N}$ is an algebraic subset of $C^{n}$, which is closed and $G^{c}$-invariant, owing to the $G^{c}$-covariance of $\partial f\left(z^{\prime}\right)$. The set $\mathscr{N}$ contains therefore a closed $G^{c}$-orbit (the closure of every $G^{c}$-orbit intersecting $\mathscr{N}$ is contained in $\mathscr{N}$ ). By Proposition 3.1, on each closed $G^{c}$-orbit $\Omega \subset \mathscr{N}$ the $D$-term vanishes at all the points of a unique $G$-orbit, formed by all the vectors of minimum length.

This completes the proof of Proposition 3.3 which, through the HilbertMumford theorem (see P3), can also be formulated in the following way:

Proposition 3.4. Let $\bar{z}$ be a zero of $\partial f\left(z^{\prime}\right)$. Then there exists a one-parameter subgroup of $G^{c},\{\exp (x \tau)\}_{x \in R}$, such that the limit $z=\lim _{x \rightarrow \infty} \exp (x \tau) \cdot \bar{z}$ is finite and $D\left(z, z^{*}\right)=0$.

The arguments used to prove Proposition 3.3 can be extended to the case of spontaneous supersymmetry breaking when its direction $\partial f(z) \neq 0$ is invariant by $G$.

Proposition 3.5. If the vector $\varphi$, which equals the gradient of the superpotential at a point $\bar{z}$ where the $F$-term takes on its minimum, is invariant by $G$, at every point where $V\left(z^{\prime}, z^{\prime *}\right)$ takes on its minimum the D-term vanishes.

Proof. The (closed) algebraic set $I=\left\{z^{\prime} \in C^{n} \mid \partial f\left(z^{\prime}\right)=\varphi\right\}$ is $G^{c}$-invariant and therefore contains a closed $G^{c}$-orbit $\Omega$. According to Proposition 3.1, on $\Omega$ there are points where the $D$-term vanishes and the potential takes on its minimum.

In general, there seems to be no reason why the minima of the potential should correspond also to stationary points of its $F$ and $D$ components separately. However, when this happens, some general conclusions can be drawn. In fact, the quadratic structure and the homogeneity in $z^{\prime}$ and $z^{\prime *}$ of the $D$-term imply a one-toone correspondence between its zeroes and stationary points. Thus, using also Propositions 3.1 and 2.1, it is straightforward to prove the following statement:

Proposition 3.6. Every stationary point $z$ of the potential which is also a stationary point of its $F$-term is a zero of the D-term, so that the $G^{c}$-orbit $\Omega$ through z is closed and $G_{z}^{c}=G_{z}{ }^{c}$. Moreover, the function $\left.F\right|_{\Omega}$ takes on its minimum at $z$ and $G_{\varphi}{ }^{c}=G_{\varphi}^{c}$, for $\varphi=\partial f(z)$.

It would also be easy to show that, if $z$ is a stationary point of the potential, then it will be a stationary point of $\left.F\right|_{G^{c} \cdot z}$ if and only if it is a zero of the $D$-term. This means that the points, if any, at which the potential takes on its minimum and $D$ is different from zero do not correspond to minima even of the restriction of $F$ to the $G^{c}$-orbit.

Propositions 3.1, 3.3, and 3.5 yield a good characterization of the vacuum vector $z$ when supersymmetry is not broken or broken in a direction which is 
invariant by $G$. In these cases a comparison with the results obtained in Sect. 2 shows that the gauging of the internal symmetry shifts all the points where the potential takes on its minimum to those points $z$ of convenient closed $G^{c}$-orbits which are at minimum distance from the origin. As a consequence $G_{z}^{c}$ becomes locally maximal and all the degeneracies of the vacuum state which originate from the $G^{c}$-invariance of the superpotential are removed, but for the degeneracies along the $G$-orbit through $z$.

\section{Supersymmetric Theories with a Partially Gauged Internal Symmetry}

If only a subgroup $G^{\prime}$ of the internal symmetry group $G$ is gauged, the invariance of the $D$-term with respect to transformations of $G$ sets severe constraints on the way $G^{\prime}$ is imbedded in $G$. A sufficient condition for the invariance of the $D$-term would be, for instance, that $G^{\prime}$ is an invariant subgroup of $G$, in which case the compactness of $G$ implies that $G=G^{\prime} \otimes G^{\prime \prime}$.

By the same arguments we have used in the case of complete gauging of $G$, we can conclude that, also in the case of partially gauged internal symmetry, the $D$ term vanishes in correspondence with an absolute minimum of the potential when the $F$-term takes on its minimum at a point where the gradient of the superpotential is invariant by $G^{\prime}$. The $D$-term vanishes also at points where the potential takes on its minimum and the $F$-term is stationary.

\section{Appendix A}

In this appendix we shall prove some of the mathematical results stated in part I of the paper, starting from the statements under items P6 and P8 of Sect. I.2.

To this purpose let us define the following real positive semidefinite function on $G^{c}$ :

$$
\varrho_{z}(\gamma)=\|\gamma \cdot z\|^{2}, \quad \gamma \in G^{c}
$$

Only in the aim of simplifying our arguments, we shall assume that in the representation of $\mathscr{G}$ we shall be concerned with, all the elements of a given Cartan subalgebra $\mathscr{H}$ of $\mathscr{G}$ are diagonal matrices. We shall denote by $\tilde{\mathscr{H}}$ the real Lie subalgebra $i \mathscr{H}$ and by $\tilde{H}$ the abelian real subgroup of $G^{c}$ formed by all the elements $d=\exp h$, with $h \in \tilde{\mathscr{H}}$.

Lemma 1 (Cartan Decomposition). Every element $\gamma \in G_{0}^{c}$ can be decomposed in the following form: $\gamma=g d g^{\prime}$, where $g$ and $g^{\prime}$ are convenient elements of $G_{0}$ and $d \in \tilde{H}$.

Proof. Let $\gamma \in G_{0}^{c}$, then there exists $\tau \in \mathscr{G}^{c}$ such that:

$$
\gamma=\exp \tau, \quad \tau=t_{1}+i t_{2} ; \quad t_{1}, t_{2} \in \mathscr{G} .
$$

Since $\gamma^{+}=\exp \left(-t_{1}+i t_{2}\right) \in G_{0}^{c}$, the matrix $\gamma \gamma^{+}$is a positive definite element of $G_{0}^{c}$. Then it can be written in the form:

$$
\gamma \gamma^{+}=\exp (i t), \quad t \in \mathscr{G},
$$


and diagonalized by means of a unitary transformation induced by an element $g$ of $G_{0}$ :

$$
g^{-1}\left(\gamma \gamma^{+}\right) g=d^{2} \in \tilde{H}
$$

If $g^{\prime} \in G_{0}^{c}$ is defined through the following relation:

$$
\gamma=g d g^{\prime} \in G_{0}^{c}
$$

it turns out to be unitary:

$$
g^{+} g^{\prime}=\gamma^{+} g d^{-2} g^{+} \gamma=\gamma^{+}\left(\gamma \gamma^{+}\right)^{-1} \gamma=1,
$$

and therefore $g^{\prime} \in G_{0}$. q.e.d.

Lemma 2. For every $h \in \tilde{\mathscr{H}}$, the real function $f_{h}(\lambda)$ :

$$
f_{h}(\lambda)=\varrho_{z}(\exp \lambda h), \quad \lambda \in R^{1},
$$

has positive second order derivative, unless $h \cdot z=0$, in which case $f_{h}$ is a constant. Proof. If $h \cdot z=0$, then $\exp (\lambda h) \cdot z=z$, so that $f_{h}(\lambda)=\|z\|^{2}=$ const. Thus we have only to prove that $f_{h}^{\prime \prime}(\lambda)>0$ for all values of $\lambda$, whenever $h \cdot z \neq 0$. This follows from a trivial direct calculation:

$$
f_{h}^{\prime \prime}(\lambda)=\sum_{1}^{n} h_{i i}^{2}\left|z_{i}\right|^{2} \exp \left(2 \lambda h_{i i}\right)>0 .
$$

Recalling that every real function defined on $R^{1}$, with an everywhere positive second derivative, has a minimum if and only if it has a stationary point and that it has at most one stationary point, it will be easy to prove the following lemma:

Lemma 3. Let $\left.\varrho_{z}\right|_{\tilde{H}}$ denote the restriction of $\varrho_{z}$ to $\tilde{H} \subset G^{c}$. Then there is a one-to-one correspondence between the points where $\left.\varrho_{z}\right|_{\tilde{H}}$ takes on its minimum and its stationary points. Moreover, if $\left.\varrho_{z}\right|_{\tilde{H}}$ has a stationary point at $\gamma=1$, then the only points of $\tilde{H}$ where it takes on its minimum are the elements of the stability subgroup of $\widetilde{H}$ at $z, \tilde{H}_{z}$.

Proof. The Lie group structure of $\tilde{H}$ assures that the absolute minimum of $\left.\varrho_{z}\right|_{\tilde{H}}$, when it exists, is a local minimum. We shall show that, vice versa, at every stationary point the function $\left.\varrho_{z}\right|_{\tilde{H}}$ takes on its absolute minimum.

Let us assume that $\left.\varrho_{z}\right|_{\tilde{H}}$ has a stationary point at $d_{0}$ in $\tilde{H}$. Since $\varrho_{z}(d)=\varrho_{d \cdot z}(1)$ and the left translation is an analytical transformation in a Lie group, by changing if necessary the choice of $z$ ( $z$ is arbitrary), it will not be restrictive to assume that $d_{0}=1$. We shall denote by $d=\exp h, h \in \tilde{\mathscr{H}}$, a general element of $\tilde{H}$. In our assumptions the function $f_{h}(\lambda)$ :

$$
f_{h}(\lambda)=\varrho_{z}(\exp \lambda h), \quad \lambda \in R^{1},
$$

has a stationary point at $\lambda=0$. Therefore, from Lemma 2 we obtain:

$$
\varrho_{z}(1)=f_{h}(0) \leqq f_{h}(\exp h)=\varrho_{z}(d),
$$

and the equality sign holds if and only if $d \in \tilde{H}_{z}$.

Lemma 3 can be restated in the following form: The function $\left.\|z\|^{2}\right|_{\Omega}$, defined on the $\widetilde{H}$-orbit $\Omega$, has at most one stationary point, where it takes possibly its absolute minimum. 
In terms of the function $\varrho_{z}(\gamma), \gamma \in G^{c}$, with the help of Lemma 1 we obtain:

Lemma 4. There is a one-to-one correspondence between the points where $\varrho_{z}$ takes on its minimum and its stationary points. Moreover, if $\varrho_{z}$ has a stationary point at $\gamma=1$, then all the vectors of minimum length on the orbit $G^{c} \cdot z$ form the orbit $G \cdot z$.

Proof. Owing to the Lie group structure of $G^{c}$, the function $\varrho_{z}(\gamma)$ takes on its minimum at stationary points.

In order to prove the converse, it will not be restrictive to assume that $\varrho_{z}(\gamma)$ has a stationary point at the neutral element 1 of $G^{c}$, just as was done in the proof of Lemma 3. According to Lemma 1, a general element $\gamma \in G_{0}^{c}$ can be factorized in the following way:

$$
\gamma=g d g^{\prime}, \quad g, g^{\prime} \in G_{0}, \quad d \in \tilde{H}
$$

therefore, using also the unitarity of $g$, we obtain:

$$
\varrho_{z}(\gamma)=\varrho_{z}\left(g d g^{\prime}\right)=\varrho_{g^{\prime} \cdot z}(d)
$$

Since the right and left translations are analytical operations in a Lie group, the function $\varrho_{g^{\prime} \cdot z}(d)$, considered as a function on $\tilde{H}$, has a stationary point at $d=1$, in our assumptions. Using Lemma 3 and the unitarity of $g \in G$, we can therefore conclude that:

$$
\varrho_{z}(\gamma)=\varrho_{g^{\prime} \cdot z}(d) \leqq \varrho_{g^{\prime} \in z}(1)=\varrho_{z}(1),
$$

where the equality sign holds if and only if $d \in \widetilde{H}_{g^{\prime} \cdot z}$, i.e. if and only if $\gamma \cdot z=\left(g g^{\prime}\right) \cdot z$, so that $\gamma \cdot z \in G \cdot z$.

The first part of Lemma 4 coincides with P6, the second one allows us to conclude like in P8.

Proof of P7. If the $G^{c}$-orbit $\Omega$ is closed, the continuous function $\left.\|z\|^{2}\right|_{\Omega}$, which is bounded below, has a minimum. Vice versa, let us assume that $\left.\|z\|^{2}\right|_{\Omega}$ takes on its minimum for $z=z_{0}$. We shall prove that the following limit:

$$
\lim _{\lambda \rightarrow \infty} \exp (i \lambda t) \cdot z_{0}, \quad t \in \mathscr{G}
$$

equals $z_{0}$, if it exists, so that the $G^{c}$-orbit through $z_{0}$ is closed according to the Hilbert-Mumford criterium (see P3).

Whichever is $t \in \mathscr{G}$, there exists $k \in G$ such that:

$$
k t k^{-1}=h \in \tilde{\mathscr{H}} \text {. }
$$

In correspondence let us define:

$$
f_{h}=\left\|\exp (i \lambda t) \cdot z_{0}\right\|^{2}=\varrho_{k \cdot z_{0}}(\exp \lambda h) .
$$

Then, in our assumptions, the function $f_{h}(\lambda)$ has a stationary point at $\lambda=0$ (see Lemma 3), so that $f_{h}^{\prime}(0)=0$ and, as shown in Lemma 2:

i) if $h \cdot\left(k \cdot z_{0}\right)=0$, then $f_{h}(\lambda)=$ const;

ii) if $h \cdot\left(k \cdot z_{0}\right) \neq 0$, then $f_{h}^{\prime \prime}(\lambda)>0$.

In the first case the limit in Eq. (A.13) exists, but equals $z_{0}$. In the second case the limit diverges, as a consequence of the relations $f_{h}^{\prime}(0)=0$ and $f_{h}^{\prime \prime}(\lambda)>0$, for all $\lambda$ 's. 
Proof of P9. The statement in P9 can be derived as a simple consequence of Eq. (I.3.6) [3]. In fact, let $z$ be a vector of minimum length on its $G^{c}$-orbit, so that Eq. (I.3.9) holds. If $\tau \in \mathscr{G}^{c}$ and $\tau=t+i t^{\prime}$, where $t, t^{\prime} \in \mathscr{G}$, then $0=\tau \cdot \zeta=t \cdot \zeta+\varepsilon t^{\prime} \cdot \zeta$. Since $t \cdot \zeta$ and $\varepsilon t^{\prime} \cdot \zeta$ are orthogonal vectors, they must vanish separately. This implies: $t, t^{\prime} \in \mathscr{G}_{z}$, or equivalently: $\mathscr{G}^{c}{ }_{z}=\mathscr{G}_{z}{ }^{c}$.

An equivalent proof of $\mathbf{P 9}$, which sheds some light on the physical meaning of the condition $\mathscr{G}_{z}^{c}=\mathscr{G}_{z}{ }^{c}$, is the following. Suppose $D_{\alpha}\left(z, z^{*}\right)=\left(z, t_{\alpha} z\right)=0, \alpha=1, \ldots, r$. Then the positive semidefinite matrix $K_{\alpha \beta}=\left(t_{\alpha} z, t_{\beta} z\right)$, which in globally supersymmetric gauge theories is related to the mass matrix of the spin $\frac{1}{2}$ fermions, turns out to be real and symmetric, so that it admits a complete set of real eigenvectors. In particular, all the vectors of its null space can be written in the form $w=u+i v$, where $u$ and $v$ are real vectors and belong to the null space of $K$. Now, if $\tau=\sum_{1}^{r} w_{\alpha} t_{\alpha}$ and $\tau \in \mathscr{G}^{c}$, then the vector $w=\left(w_{1}, \ldots, w_{r}\right)$ must be in the null space of $K$. Since $K$ is a real matrix, the same must be true of the real and imaginary parts $u$ and $v$ of $w$. Therefore, $\tau=t+i t^{\prime}$, where $t=\sum_{1}^{r} u_{\alpha} t_{\alpha}$ and $t^{\prime}=\sum_{1}^{r} v_{\alpha} t_{\alpha}$ both belong to $\mathscr{G}_{z}$.

\section{References}

1. We refer to two recent books: Wess, J., Bagger, J.: Supersymmetry and supergravity. Princeton, NJ: Princeton University Press 1983

Gates, G., Jr., Grisaru, M.T., Roček, M., Siegel, W.: Superspace. Reading, MA: Benjamin Cummings 1983

2. See, for instance, Zumino, B.: Int. Conf. on high energy physics (Lisbon, July 1981), CERN preprint 3167, LBL preprint 13691 and references therein

Ovrut, B., Wess, J.: Supersymmetry $R_{\xi}$ gauge and radiative symmetry breaking. Phys. Rev. D 25, 409 (1982)

Bagger, J., Wess, J.: Op cit.

3. Gatto, R., Sartori, G.: Gauge symmetry breaking in supersymmetric gauge theories: Necessary and sufficient condition. Phys. Lett. 118 B, 79 (1982), and Zeros of the $D$-term and complexification of the gauge group in supersymmetric theories. $157 \mathrm{~B}, 389$ (1985)

4. Girardi, G., Sorba, P., Stora, R.: Comments on the spontaneous symmetry breaking in supersymmetric theories. Phys. Lett. 144 B, 212 (1984)

5. Procesi, C., Schwarz, G.W.: The geometry of orbit spaces and gauge symmetry breaking in supersymmetric gauge theories. Phys. Lett. $161 \mathrm{~B}, 117$ (1985)

6. Borel, A.: Linear algebraic groups. New York: Benjamin 1969

7. Humphreys, J.G.: Linear algebraic groups. Graduate texts in mathematics, Vol. 21. Berlin, Heidelberg, New York: Springer 1975

8. Fogarty, J.: Invariant theory. New York: Benjamin 1969

9. Mumford, D.: Geometric invariant theory. Erg. Math., Bd. 34. Berlin, Heidelberg, New York: Springer 1965

10. Buchmüller, W., Peccei, R.D., Yanagida, T.: Quasi Nambu-Goldstone fermions. Nucl. Phys. B 227, 503 (1983), and references therein

Barbieri, R., Masiero, A., Veneziano, G.: Hierarchy of fermion masses in supersymmetric composite models. Phys. Lett. 128 B, 179 (1983)

Lerche, W.: On Goldstone fields in supersymmetric theories. Nucl. Phys. B 238, 582 (1984) Sharatchandra, H.S.: Max-Planck-Institute preprint MPI-PAE/Pth 2/84

Kugo, T., Ojima, I., Yanagida, T.: Superpotential symmetries and pseudo Nambu-Goldstone supermultiplets. Phys. Lett. 135 B, 402 (1984) 
Bando, M., Kuramoto, T., Maskawa, T., Uehara, S.: Structure of non-linear realization in supersymmetric theories. Phys. Lett. 138 B, 94 (1984)

11. Lee, C., Sharatchandra, H.S.: Max-Planck-Institute preprint PAE/Pth 54/83

Lerche, W.: See [10]

Bando, M., Kuramoto, T., Maskawa, T., Uehara, S.: See [10]

Shore, G.: Generalizations of Dashen's formula in supersymmetric QCD. Nucl. Phys. B 231, 139 (1984), and Supersymmetric Higgs mechanism with non-doubled Goldstone bosons. B 248, 123 (1984)

Goiti, J.L.: Mass generation in theories with quasi-Goldstone fermions. Nucl. Phys. B 261, 66 (1985)

12. Kempf, G., Ness, L.: In: Algebraic geometry. Lecture Notes in Mathematics, Vol. 732. Berlin, Heidelberg, New York: Springer 1978

13. Matsushima, Y.: Nagoya Math. J. 16, 215 (1960)

14. Luna, D.: Slices étales. Bull. Soc. Math. France, Mémoire 33, 81 (1973)

15. Abud, M., Sartori, G.: The geometry of orbit-space and natural minima of Higgs potentials. Phys. Lett. 104 B, 147 (1981), and The geometry of spontaneous symmetry breaking. Ann. Phys. 150, 307 (1983)

16. Schwarz, G.W.: Lifting smooth homotopies of orbit spaces. Inst. Hautes Etudes Sci. Publ. Math. (1980)

17. Gatto, R., Sartori, G.: Relies of supersymmetry in the mass spectrum after spontaneous breaking. Int. J. Mod. Phys. A 1, 683 (1986)

18. Zumino, B.: In: Unified theories of elementary particles. Breitenlohner, P., Dürr, H.P. (eds.). Berlin, Heidelberg, New York: Springer 1982

19. Fayet, P., Iliopoulos, J.: Spontaneously broken supergauge symmetries and Goldstone spinors. Phys. Lett. 51 B, 461 (1974)

20. Buccella, F., Derendinger, J.P., Ferrara, S., Savoy, C.A.: Patterns of symmetry breaking in supersymmetric gauge theories. Phys. Lett. 115 B, 375 (1982)

21. Luna, D.: Invent. Math. 29, 231 (1975)

22. Sartori, G.: A theorem on orbit structures (strata) of compact linear Lie groups. J. Math. Phys. 24, 765 (1983)

Communicated by L. Alvarez-Gaumé

Received August 1, 1986; in revised form October 2, 1986 